\title{
MODEL SPACES AND TOEPLITZ KERNELS IN REFLEXIVE HARDY SPACE
}

\author{
M. C. CÂmara, M. T. Malheiro and J. R. Partington
}

Abstract. This paper considers model spaces in an $H_{p}$ setting. The existence of unbounded functions and the characterisation of maximal functions in a model space are studied, and decomposition results for Toeplitz kernels, in terms of model spaces, are established.

Mathematics subject classification (2010): 47B35, 30H10.

Keywords and phrases: Model space, Toeplitz operator, Hardy space, near invariance.

\section{REFERENCES}

[1] C. Benhida, M. C. CÂmara And C. Diogo, Some properties of the kernel and the cokernel of Toeplitz operators with matrix symbols, Linear Algebra Appl., 432, 1 (2010), 307-317.

[2] M. C. CÂmara And J. R. Partington, Near invariance and kernels of Toeplitz operators, Journal d'Analyse Math., 124, (2014), 235-260.

[3] P. L. Duren, Theory of $H^{p}$ spaces, Dover, New York, 2000.

[4] K. M. DYAKONOv, Moduli and arguments of analytic functions from subspaces in $H^{p}$ that are invariant under the backward shift operator (Russian), Sibirsk. Mat. Zh., 31, 6 (1990), 64-79; translation in Siberian Math. J., 31, 6 (1990), 926-939 (1991).

[5] K. M. Dyakonov, Entire functions of exponential type and model subspaces in $H^{p}$ (Russian), Zap. Nauchn. Sem. Leningrad. Otdel. Mat. Inst. Steklov. (LOMI), 190, (1991), Issled. po Linein. Oper. i Teor. Funktsii., 19, 81-100, 186; translation in J. Math. Sci., 71, 1, (1994), 2222-2233.

[6] K. M. Dyakonov, Continuous and compact embeddings between star-invariant subspaces, Complex analysis, operators, and related topics, 65-76, Oper. Theory Adv. Appl., 113, Birkhäuser, Basel, 2000.

[7] K. M. DYAKONOv, Kernels of Toeplitz operators via Bourgain's factorization theorem, J. Functional Analysis, 170, (2000), 93-106.

[8] O. Frostman, Sur les produits de Blaschke, Kungl. Fysiogr. Sälsk. i Lund Förh., 12, (1942), 169_ 182.

[9] T. W. Gamelin, Uniform algebras, Second edition, American Mathematical Society, 2006.

[10] S. GARCIA AND W. T. Ross, Model spaces: a survey. Invariant subspaces of the shift operator, 197-245, Contemp. Math., 638, Amer. Math. Soc., Providence, RI, 2015.

[11] E. HAYASHI, The kernel of a Toeplitz operator, Integral Equations Operator Theory, 9, 4 (1986), 588-591.

[12] E. HaYAshi, Classification of nearly invariant subspaces of the backward shift, Proc. Amer. Math. Soc., 110, 2 (1990), 441-448.

[13] D. HitT, Invariant subspaces of $\mathscr{H}^{2}$ of an annulus, Pacific J. Math., 134, 1 (1988), 101-120.

[14] B. JacoB, J. R. PARtington And S. Pott, Weighted interpolation in Paley-Wiener spaces and finite-time controllability, Journal of Functional Analysis, 259, (2010), 2424-2436.

[15] B. JaCOB And H. Zwart, Properties of the realization of inner functions, Math. Control Signals Systems, 15, 4 (2002), 356-379.

[16] J. R. Partington, An introduction to Hankel operators, London Mathematical Society Student Texts, 13, Cambridge University Press, Cambridge, 1988. 
[17] N. K. NiKOLSKI, Operators, functions, and systems: an easy reading, Vol. 1, Mathematical Surveys and Monographs, vol. 92, American Mathematical Society, Providence, RI, 2002.

[18] D. SaRASOn, Nearly invariant subspaces of the backward shift, Contributions to operator theory and its applications (Mesa, AZ, 1987), 481-493, Oper. Theory Adv. Appl., 35, Birkhäuser, Basel, 1988.

[19] D. SARASOn, Kernels of Toeplitz operators, Toeplitz operators and related topics (Santa Cruz, CA, 1992), 153-164, Oper. Theory Adv. Appl., 71, Birkhäuser, Basel, 1994. 